\title{
Nonlinear Free Vibration Analysis of Axisymmetric Polar Orthotropic Circular Membranes under the Fixed Boundary Condition
}

\author{
Zhoulian Zheng, ${ }^{1,2}$ Jianjun Guo, ${ }^{3}$ Weiju Song, ${ }^{2}$ Xiaoting He, ${ }^{2}$ \\ Faming Lu, ${ }^{2}$ Chuanxi Xie, ${ }^{2}$ and Junyi Sun ${ }^{2}$ \\ ${ }^{1}$ Department of Civil Engineering, Chongqing University, Chongqing 400045, China \\ ${ }^{2}$ Chongqing Vocational College of Architectural Engineering, Chongqing 400039, China \\ ${ }^{3}$ Chongqing Water Resources and Electric Engineering College, Chongqing 402160, China \\ Correspondence should be addressed to Jianjun Guo; guofuzhi@126.com
}

Received 26 August 2013; Revised 20 December 2013; Accepted 15 January 2014; Published 25 February 2014

Academic Editor: Trung Nguyen Thoi

Copyright (c) 2014 Zhoulian Zheng et al. This is an open access article distributed under the Creative Commons Attribution License, which permits unrestricted use, distribution, and reproduction in any medium, provided the original work is properly cited.

\begin{abstract}
This paper presents the nonlinear free vibration analysis of axisymmetric polar orthotropic circular membrane, based on the large deflection theory of membrane and the principle of virtual displacement. We have derived the governing equations of nonlinear free vibration of circular membrane and solved them by the Galerkin method and the Bessel function to obtain the generally exact formula of nonlinear vibration frequency of circular membrane with outer edges fixed. The formula could be degraded into the solution from small deflection vibration; thus, its correctness has been verified. Finally, the paper gives the computational examples and comparative analysis with the other solution. The frequency is enlarged with the increase of the initial displacement, and the larger the initial displacement is, the larger the effect on the frequency is, and vice versa. When the initial displacement approaches zero, the result is consistent with that obtained on the basis of the small deflection theory. Results obtained from this paper provide the accurate theory for the measurement of the pretension of polar orthotropic composite materials by frequency method and some theoretical basis for the research of the dynamic response of membrane structure.
\end{abstract}

\section{Introduction}

The membrane structure is a kind of large span flexible space structure, which has a wide range of applications as discussed elsewhere [1]. However, due to its small weight and stiffness and low natural frequency as well as small damping, it is very sensitive to the action of wind, rain, and other external loads and easy to produce vibration as discussed elsewhere [2]. The deformation of membrane structure, caused by the vibration, leads to the destruction of membrane structure in practical engineering. Since this kind of case is common, studying the vibration of membrane structure and its dynamic response under the external excitation becomes the key scientific issues in the field of membrane structure. In actual engineering, the dynamic characteristics of the membrane structure mainly show up as the vibration problem in large deflection, when the membrane is under impact loading.
From the last century, many scholars at home and abroad have done a lot of research work about it. The problem of large deflection of elastic circular membrane was studied by Chien et al. [3]. A nonlinear relaxation method was employed to solve the nonlinear partial differential equations governing the large deflection response of various axisymmetric circular membranes, by Kao and Perrone [4]. The problem of large deflections of rotationally orthotropic circular membrane was investigated by Cheng and Yang [5], in which the relations between the load and membrane forces as well as the deflection and the central deflection were obtained. The large deformation of circular membrane under the concentrated force was analyzed by Chen and Zheng [6]. Kang [7] dealt with a composite rectangular membrane with an oblique interface. Young et al. [8] made a numerical calculation and experimental study on the dynamic characteristics of thin-film membranes. The nonlinear vibration analysis of 
a prestretched hyperelastic annular membrane under finite deformations was completed by Soares and Gonalves [9]. Qian [10] researched the vibration problem of the rectangular membrane with two different directions of force, circular membrane, and elliptic membrane and obtained the approximate solution of the vibration frequency of arbitrary shape membrane. Wu [11] obtained the analytical solution of natural vibration modes of the intermediate support membrane by the Laplace transform. Lin and Chen [12] used analytical method to obtain the theoretical solution of free vibration of annular isotropic membrane. Ou yang [13] studied a Robin problem of the vibration of a perturbed boundary membrane, using parametric deformation method and the condition for the solvability of the problem. The nondegenerate vibrational frequency and vibration mode of this problem are obtained. Lin and Chen [14] used the finite element method to analyze the free vibration of flat membrane. Qiao et al. [15] used the Newmark method of dynamic analysis and the NewtonRaphson iterative method of nonlinear analysis to program composition for the nonlinear dynamic response analysis of membrane structure. However, the theoretical research on the nonlinear vibration of orthotropic circular membrane is relatively rare.

In this paper, according to the large deflection theory of membrane, we study the vibration of axisymmetric polar orthotropic circular membrane and use the principle of virtual displacement to create the governing vibration equations of circular membrane with outer edges fixed. The Bessel function and the Galerkin method are used to get a generally accurate expression of the nonlinear free vibration frequency of circular membrane, and the results obtained in this paper are compared with other existing results. The work presented in this paper provides a theoretical basis for the study of the dynamic response of polar orthotropic membrane materials.

\section{Basic Equations}

The studied circular membrane is axisymmetric polar orthotropic and it is assumed that all outer edges of the membrane are fixed. $r$ denotes the radius of the membrane, $h$ denotes the thickness of the membrane, $\rho$ denotes aerial density of the membrane material, and $N_{r}$ and $N_{\theta}$ denote initial tension along radial and circumferential direction, respectively, as shown in Figure 1.

According to the large deflection theory of membrane, the equations are established, which are based on the hypothesis as follows.

(i) The membrane is soft and springy, which cannot resist moment, and the tension is in the tangent plane at any time.

(ii) There is only transverse vibration without considering the damping in vibration process.

(iii) The influence of membranes thickness variation in vibration process can be neglected.

(iv) The paper only considers geometric nonlinearity for membranes with the hypothesis that the membrane is homogeneous, continuous, and linearly elastic in vibration process.

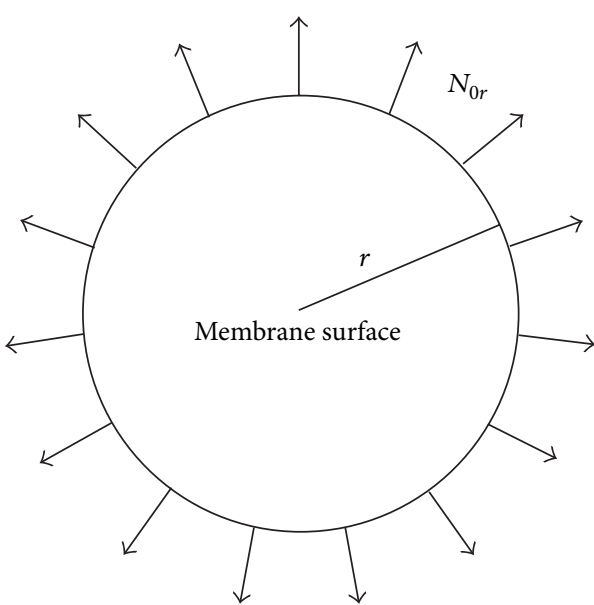

FIGURE 1: Circular membrane with all edges fixed and force diagram of the element.

The corresponding boundary conditions can be expressed as follows:

$$
\left.w\right|_{r=a}=0,\left.\quad u_{r}\right|_{r=a}=0,
$$

where $w$ denotes the deflection of membranes and $u_{r}$ denotes the radial displacement.

The geometrical equation of axisymmetric deformation of the circular membrane in polar coordinates is given by

$$
\varepsilon_{r}=\frac{d u}{d r}+\frac{1}{2}\left(\frac{d w}{d r}\right)^{2}, \quad \varepsilon_{\theta}=\frac{u}{r}
$$

The physical equations are

$$
\begin{aligned}
& N_{r}=h \sigma_{r}=\frac{E_{r} h}{1-\mu_{r} \mu_{\theta}}\left(\varepsilon_{r}+\mu_{\theta} \varepsilon_{\theta}\right), \\
& N_{\theta}=h \sigma_{\theta}=\frac{E_{\theta} h}{1-\mu_{r} \mu_{\theta}}\left(\varepsilon_{\theta}+\mu_{r} \varepsilon_{r}\right),
\end{aligned}
$$

where $E_{r}$ and $E_{\theta}$ represent Young's modulus along radial and circumferential direction, respectively; $\mu_{r} \mu_{\theta}$ represents Poisson's ratio, respectively; $\varepsilon$ represents the strain; $\sigma$ represents the stress.

The equation of compatibility is

$$
\varepsilon_{r}=\frac{d}{d r}\left(r \varepsilon_{\theta}\right)+\frac{1}{2}\left(\frac{d w(r, t)}{d r}\right)^{2}
$$

and $\varepsilon_{r}$ and $\varepsilon_{\theta}$ can be transformed into the below form, with (3); that is,

$$
\varepsilon_{r}=\frac{N_{r}}{E_{r} h}-\frac{N_{\theta} \mu_{\theta}}{E_{\theta} h}, \quad \varepsilon_{\theta}=\frac{N_{\theta}}{E_{\theta} h}-\frac{N_{r} \mu_{r}}{E_{r} h} .
$$
yield

Substituting (5) into (4) and letting $\lambda^{2}=E_{\theta} / E_{r}=\mu_{\theta} / \mu_{r}$

$$
r \frac{d^{2}}{d r^{2}}\left(r N_{r}\right)+\frac{d\left(r N_{r}\right)}{d r}-\lambda^{2} N_{r}=-\frac{1}{2} E_{\theta} h\left(\frac{d w}{d r}\right)^{2} .
$$


Introducing the stress function $\sigma_{r}=(1 / r)(d \phi / d r), \sigma_{\theta}=$ $d^{2} \phi / d r^{2}$ and letting $N_{r}=h \sigma_{r}=(h / r)(d \phi / d r), N_{\theta}=h \sigma_{\theta}=$ $h\left(d^{2} \phi / d r^{2}\right),(6)$ can be simplified as follows:

$$
r \frac{d^{3} \phi}{d r^{3}}+\frac{d^{2} \phi}{d r^{2}}-\lambda^{2} \frac{1}{r} \frac{d \phi}{d r}=-\frac{1}{2} E_{\theta}\left(\frac{d w(r, t)}{d r}\right)^{2} .
$$

According to the principle of virtual displacement, considering the vibration inertia power of membranes as distributed load, the equation of the large deflection vibration of membranes can be given as follows:

$$
\int_{0}^{a}\left[\psi+h\left(\sigma_{r}+\sigma_{0 r}\right) \frac{\partial w}{\partial r}\right] \frac{\partial \delta w}{\partial r} r d r=0,
$$

where $\sigma_{0 r}$ denotes pretension of circular membrane; load function is $\psi=(1 / r) \int_{0}^{r}(-\rho h \ddot{w}) r d r$.

Taking the small deflection theory of membrane into account, every piece element of membranes has no elongation; therefore, according to the elastic Hooke's law, the membrane is given a constant tension. The equation of the small deflection vibration of membranes should be

$$
\int_{0}^{a}\left(\psi+h \sigma_{0 r} \frac{\partial w}{\partial r}\right) \frac{\partial \delta w}{\partial r} r d r=0 .
$$

\section{Governing Equation}

The equation of transverse free vibration of membranes is

$$
\nabla^{2} w=\frac{\rho}{N} \frac{\partial^{2} w}{\partial t^{2}}
$$

where $w$ denotes the deflection of membranes. On the polar coordinates, $\nabla^{2}=\partial^{2} / \partial r^{2}+(1 / r)(\partial / \partial r)+\left(1 / r^{2}\right)\left(\partial^{2} / \partial \theta^{2}\right)$; for the axisymmetric circular membrane, $\nabla^{2}=d^{2} / d r^{2}+$ $(1 / r)(d / d r)$.

In order to solve the vibration differential equation, through the method of separation of variables, the functions which satisfy the boundary conditions (1) are taken as follows:

$$
w(r, t)=W(r) T(t) .
$$

Substituting (11) into (6) yields

$$
\nabla^{2} W \cdot T(t)=\frac{\rho}{N} \cdot \ddot{T}(t) \cdot W .
$$

Letting $\mu^{2}=\rho \ddot{T}(t) / N T(t)$, then

$$
\nabla^{2} W=\frac{\rho}{N} \mu^{2} W
$$

Letting $(\rho / N) \mu^{2}=\gamma^{2}$, then

$$
\begin{gathered}
\left(\nabla^{2}-\gamma^{2}\right) W=0 \\
\left(\frac{\partial^{2}}{\partial r^{2}}+\frac{1}{r} \frac{\partial}{\partial r}-\gamma^{2}\right) W=0 .
\end{gathered}
$$

Let the shape function of displacement be

$$
W=R(r) \cos n \theta,
$$

where $n=0,1,2,3, \ldots$. When $n=0$, the shape function is symmetrical. In this paper, the deformation of circular membranes is axisymmetric, so $n=0$.

Substituting $W=R(r) \cos n \theta$ into (15), an ordinary differential equation is obtained:

$$
\frac{d^{2} R}{d r^{2}}+\frac{1}{r} \frac{d R}{d r}+\left(\gamma^{2}-\frac{n^{2}}{r^{2}}\right) R=0 .
$$

A dimensionless parameter $(x=\gamma r)$ is introduced; we have

$$
x^{2} \frac{d^{2} R}{d r^{2}}+x \frac{d R}{d r}+\left(x^{2}-n^{2}\right) R=0 .
$$

A general solution for this differential equation is

$$
R=C_{1} J_{n}(x)+C_{2} N_{n}(x),
$$

where $J_{n}(x)$ and $N_{n}(x)$ are the first and second kind Bessel functions of the Order- $N$ real variable, respectively:

$$
\begin{aligned}
& J_{n}(x)=\sum_{k=0}^{\infty}(-1)^{k} \frac{1}{k ! \Gamma(n+k+1)}\left(\frac{x}{2}\right)^{2 k+n} \quad(|\arg z|<\pi), \\
& N_{n}(x)=\frac{J_{n}(x) \cos n \pi-J_{-n}(x)}{\sin n \pi} \quad(|z|<\infty,|\arg z|<\pi) .
\end{aligned}
$$

Equation (11) is only the general solution of the governing differential equation. Only after it satisfies concrete boundary conditions does it meet the practical engineering problems. Due to the clamped edges of the circular membrane, we have

$$
\left.w\right|_{r=a}=0, \quad|w|<\infty,\left.\quad \frac{\partial w}{\partial r}\right|_{r=a}=0,
$$

where $a$ denotes the radius of the circular membrane.

$N_{n}(x)$ and $J_{n}(x)$ are infinite. Because $|w|<\infty$, when $x=$ $\gamma r=0$, so $C_{2}=0$.

And (19) can be simplified as

$$
\begin{gathered}
R=C_{1} J_{n}\left(\gamma_{i} r\right), \\
W(r)=C_{1} J_{n}\left(\gamma_{i} r\right) \cos n \theta,
\end{gathered}
$$

And when $n=0$, the vibration mode is symmetrical. In this paper, the deformation of the circular membrane is axisymmetric, so $n=0$; namely,

$$
W(r)=C_{1} J_{0}\left(\gamma_{i} r\right) .
$$

Substituting the boundary conditions into (19) and (23) yields

$$
C_{1} J_{0}\left(\gamma_{i} a\right)=0
$$

Because $C_{1}$ is not identically equal to zero, there is $\gamma_{i}$, satisfying

$$
J_{0}\left(\gamma_{i} a\right)=0 .
$$


Namely, $\gamma_{i} a$ is the zero solution of the $J_{0}(x)$.

Using the MATLAB software to solve the six zero point of the first kind zero-order Bessel function, we have rootBessel $=2.4048,5.5201,8.6537,11.7915,14.9309,18.0711$. have

Obtaining the Bessel function expansion by definition, we

$$
J_{0}(x)=1-\frac{x^{2}}{2^{2}}+\frac{x^{4}}{2^{4} \cdot(2 !)^{2}}-\frac{x^{6}}{2^{6} \cdot(3 !)^{2}}+\cdots
$$

The front four of $J_{0}(x)$ will be used in the following, namely:

$$
J_{0}(x)=1-\frac{x^{2}}{4}+\frac{x^{4}}{64}-\frac{x^{6}}{2304}
$$

Substituting (27) into (11), the deflection expression is

$$
w(r, t)=J_{0}\left(\gamma_{i} r\right) T(t)
$$

Substituting (27) into (28) yields

$$
\begin{array}{r}
w(r, t)=\left(b+c \frac{r^{2}}{a^{2}}+d \frac{r^{4}}{a^{4}}+e \frac{r^{6}}{a^{6}}\right) T(t), \\
b, d,>0, \quad c, e<0 .
\end{array}
$$
yield

Substituting (29) into (6) and solving the Euler equation

$$
\frac{d \phi}{d r}=-\frac{E_{\theta}}{2} X T^{2}(t)+C_{1} r^{\lambda}+C_{2} r^{-\lambda}
$$

where

$$
\begin{aligned}
X= & \frac{4 c^{2}}{\left(9-\lambda^{2}\right) a^{4}} r^{3}+\frac{16 d^{2}+24 c e}{\left(49-\lambda^{2}\right) a^{8}} r^{7}+\frac{36 e^{2}}{\left(121-\lambda^{2}\right) a^{12}} r^{11} \\
& +\frac{16 c d}{\left(25-\lambda^{2}\right) a^{6}} r^{5}+\frac{48 d e}{\left(81-\lambda^{2}\right) a^{10}} r^{9}
\end{aligned}
$$

when $r=0$, the stress $\left(\sigma_{r}\right)$ is finite, so

$$
C_{2}=0
$$
yields:

At the same time, the boundary condition is: $\left.u_{r}\right|_{r=a}=0$,

$$
\begin{gathered}
\left.r \varepsilon_{\theta}\right|_{r=a}=0 \\
\left.\left(\frac{r}{E_{\theta}} \frac{d^{2} \phi}{d r^{2}}-\frac{\mu_{r}}{E_{r}} \frac{d \phi}{d r}\right)\right|_{r=a}=0
\end{gathered}
$$

Substituting (30) into (33) yields

$$
C_{1}=\frac{E_{\theta}}{2\left(\lambda-\mu_{\theta}\right) a^{\lambda-1}} Y T^{2}(t),
$$

where

$$
\begin{gathered}
Y=a^{-2}\left\{\frac{4 c^{2}\left(3-\mu_{\theta}\right)}{\left(9-\lambda^{2}\right)}+\frac{16\left(d^{2}+24 c e\right)\left(7-\mu_{\theta}\right)}{\left(49-\lambda^{2}\right)}\right. \\
+\frac{36 e^{2}\left(11-\mu_{\theta}\right)}{\left(121-\lambda^{2}\right)}+\frac{16 c d\left(5-\mu_{\theta}\right)}{\left(25-\lambda^{2}\right)} \\
\left.+\frac{48 d e\left(9-\mu_{\theta}\right)}{\left(81-\lambda^{2}\right)}\right\} .
\end{gathered}
$$

Substituting (32) and (34) into (30) yields

$$
\frac{d \phi}{d r}=\left[-\frac{E_{\theta}}{2} X+\frac{Y E_{\theta}}{2\left(\lambda-\mu_{\theta}\right) a^{\lambda-1}} r^{\lambda}\right] T^{2}(t) .
$$

Substituting (29) into (8), according to the Galerkin method, yields

$$
\int_{0}^{a} Q \frac{d W(r)}{d r} r d r=0
$$

Here, $Q=\psi+h\left((1 / r)(d \phi / d r)+\sigma_{0 r}\right)(\partial w / \partial r)$

$$
\begin{aligned}
\int_{0}^{a} \psi \frac{d W(r)}{d r} r d r & =\int_{0}^{a} \frac{1}{r} \int_{0}^{r}(-\rho h \ddot{w}) r d r \frac{d W(r)}{d r} r d r \\
& =-S a^{2} \rho h \ddot{T}(t)
\end{aligned}
$$

where

$$
\begin{aligned}
& \int_{0}^{a} h\left(\frac{1}{r} \frac{d \phi}{d r}+\sigma_{0 r}\right) \frac{\partial w}{\partial r} \frac{d W(r)}{d r} r d r \\
& =\left(-\frac{h}{2} E_{\theta} F_{1}+\frac{Y E_{\theta} h}{2\left(\lambda-\mu_{\theta}\right)} F_{2}\right) T^{3}(t)+H_{1} h \sigma_{0 r} T(t),
\end{aligned}
$$

where

$$
\begin{aligned}
F_{1} & \left\{\left(\frac{2}{3} c^{2}+\frac{8}{5} d^{2}+\frac{18}{7} e^{2}+2 c d+4 d e+\frac{12}{5} c e\right)\right. \\
& \times\left(9-\lambda^{2}\right) \\
& +\left(16 d^{2}+24 c e\right) \\
& \times\left(\frac{2}{5} c^{2}+\frac{8}{7} d^{2}+2 e^{2}+\frac{4}{3} c d+3 d e+\frac{12}{7} c e\right) \\
& \times\left(49-\lambda^{2}\right)^{-1} \\
+ & 36 e^{2}\left(\frac{2}{7} c^{2}+\frac{8}{9} d^{2}+\frac{18}{11} e^{2}\right. \\
& \left.+c d+\frac{12}{5} d e+\frac{4}{3} c e\right)
\end{aligned}
$$




$$
\begin{gathered}
\times\left(121-\lambda^{2}\right)^{-1} \\
+16 c d\left(\frac{1}{2} c^{2}+\frac{4}{3} d^{2}+\frac{9}{4} e^{2}\right. \\
\left.+\frac{8}{5} c d+\frac{24}{7} d e+2 c e\right) \\
\times\left(25-\lambda^{2}\right)^{-1} \\
+48 d e\left(\frac{1}{3} c^{2}+d^{2}+\frac{9}{5} e^{2}\right. \\
\left.+\frac{8}{7} c d+\frac{8}{3} d e+\frac{3}{2} c e\right) \\
\left.\times\left(81-\lambda^{2}\right)^{-1}\right\}, \\
F_{2}=\frac{4 c^{2}}{3+\lambda}+\frac{16 d^{2}+24 c e}{7+\lambda}+\frac{36 e^{2}}{11+\lambda}+\frac{16 c d}{5+\lambda}+\frac{48 \mathrm{de}}{9+\lambda}, \\
H_{1}=c^{2}+2 d^{2}+3 e^{2}+\frac{8}{3} c d+\frac{24}{5} d e+3 c e .
\end{gathered}
$$

Substituting all the above into (8) yields

$$
\begin{aligned}
\frac{d^{2} T(t)}{d t^{2}} & +\left(-\frac{H_{1} \sigma_{0 r}}{S a^{2} \rho}\right) T(t) \\
& +\left[\frac{E_{\theta} F_{1}}{2 S a^{2} \rho}-\frac{E_{\theta} Y}{2 S a^{2} \rho\left(\lambda-\mu_{\theta}\right)}\right] T^{3}(t)=0 .
\end{aligned}
$$

Letting $M=-H_{1} \sigma_{0 r} / S a^{2} \rho, N=E_{\theta} F_{1} / 2 S a^{2} \rho-E_{\theta} Y /$ $2 S a^{2} \rho\left(\lambda-\mu_{\theta}\right)$ yields

$$
\frac{d^{2} T(t)}{d t^{2}}+M \cdot T+N \cdot T^{3}(t)=0
$$

\section{Solution of Free Vibration Frequency}

Integrating (42) yields

$$
\left(\frac{d T(t)}{d t}\right)^{2}+M \cdot T^{2}(t)+\frac{N}{2} \cdot T^{4}(t)=K
$$

In (43), the value of $K$ is determined by the initial conditions. Assuming that the initial displacement is $\left.T\right|_{t=0}=T_{0}, T_{0}$ is the amplitude of the membrane, so the initial velocity is

$$
\left.\frac{d T(t)}{d t}\right|_{t=0}=0
$$

Substituting $\left.T\right|_{t=0}=T_{0}$ and (44) into (43) yields

$$
K=M T_{0}^{2}+\frac{N}{2} T_{0}^{4}
$$

Substituting $K=M T_{0}^{2}+(N / 2) T_{0}^{4}$ into (43) yields

$$
\begin{aligned}
\frac{d T(t)}{d t}= & T_{0}^{2} \sqrt{\frac{M}{T_{0}^{2}}+\frac{N}{2}} \\
& \cdot \sqrt{\left(1-\frac{T^{2}}{T_{0}^{2}}\right) \cdot\left(1+\frac{N T_{0}^{2}}{2 M+N T_{0}^{2}} \frac{T^{2}}{T_{0}^{2}}\right)} .
\end{aligned}
$$

Letting $\xi=T_{0}^{2} \sqrt{M / T_{0}^{2}+N / 2}, k^{2}=N T_{0}^{2} /\left(2 M+N T_{0}^{2}\right)$.

Integrating (46) by the method of separation of variables, we can obtain the period of the vibration of the membrane:

$$
Z=\frac{4}{\xi} \int_{0}^{T_{0}} \frac{1}{\sqrt{\left(1-\left(T^{2} / T_{0}^{2}\right)\right)\left(1+k^{2} \cdot\left(T^{2} / T_{0}^{2}\right)\right)}} d T .
$$

Letting $T / T_{0}=\sin \theta$, then (47) can be simplified as follows:

$$
Z=\frac{4 T_{0}}{\xi} \int_{0}^{\pi / 2} \frac{1}{\sqrt{1+k^{2} \cdot \sin ^{2} \theta}} d \theta, \quad(0 \leq k \cdot \sin \theta \leq 1),
$$

where $\left(1+k^{2} \cdot \sin ^{2} \theta\right)^{-1 / 2}$ could be spread as a power series with respect to $k \cdot \sin \theta$ :

$$
\begin{aligned}
\left(1+k^{2} \cdot \sin ^{2} \theta\right)^{-1 / 2}= & 1-\frac{1}{2} k^{2} \sin ^{2} \theta+\frac{1 \cdot 3}{2 \cdot 4} k^{4} \sin ^{4} \theta \\
& +\cdots+(-1)^{n} \frac{(2 n-1) ! !}{(2 n) ! !}(k \sin \theta)^{2 n} .
\end{aligned}
$$

Substituting (49) into (48) and then solving (48) through integrating item by item yield

$$
\begin{aligned}
& Z=\frac{4 T_{0}}{\xi} \int_{0}^{\pi / 2}\left[1-\frac{1}{2} k^{2} \sin ^{2} \theta+\frac{1 \cdot 3}{2 \cdot 4} k^{4} \sin ^{4} \theta\right.\left.+\cdots(-1)^{n} \frac{(2 n-1) ! !}{(2 n) ! !}(k \sin \theta)^{2 n}\right] d \theta \\
&=\frac{2 \pi T_{0}}{\xi}\left[1-\left(\frac{1}{2}\right)^{2} k^{2}+\left(\frac{1 \cdot 3}{2 \cdot 4}\right)^{2} k^{2}\right. \\
&\left.+\cdots+(-1)^{p}\left(\frac{(2 p-1) ! !}{(2 p) ! !}\right)^{2} k^{2 p}\right] \\
&=\frac{2 \pi T_{0}}{\xi} \sum_{n=0}^{\infty}(-1)^{p}\left(\frac{(2 p-1) ! !}{(2 p) ! !}\right)^{2} k^{2 p}
\end{aligned}
$$

where $p=n=0,1,2,3, \ldots$.

Therefore, the vibration frequency of the circular membrane is

$\omega=\frac{2 \pi}{Z}$

$$
=\frac{\sqrt{M+(N / 2) T_{0}^{2}}}{\sum_{p=0}^{\infty}(-1)^{p}((2 p-1) ! ! /(2 p) ! !)^{2}\left(N T_{0}^{2} /\left(2 M+N T_{0}^{2}\right)\right)^{p}},
$$


TABLE 1: The frequency under different initial displacements $(r=$ $0.5 \mathrm{~m}$ and $\sigma_{0 r}=5.0 \times 10^{3} \mathrm{kN} / \mathrm{m}^{2}$ ).

\begin{tabular}{lcccc}
\hline$\omega$ & $T_{0}=0.10$ & $T_{0}=0.08$ & $T_{0}=0.04$ & $T_{0}=0.01$ \\
\hline $\mathrm{Rad} / \mathrm{s}$ & 735.605 & 610.032 & 381.648 & 271.525 \\
$\mathrm{~Hz}$ & 117.075 & 97.090 & 60.741 & 43.215 \\
\hline$\omega$ & $T_{0}=0.005$ & $T_{0}=0.001$ & $T_{0}=0.0001$ & $T_{0} \rightarrow 0$ \\
\hline $\mathrm{Rad} / \mathrm{s}$ & 264.678 & 262.444 & 262.351 & 262.350 \\
$\mathrm{~Hz}$ & 42.125 & 41.769 & 41.754 & 41.754 \\
\hline
\end{tabular}

where $M=-H_{1} \sigma_{0 r} / S a^{2} \rho, N=E_{\theta} F_{1} / 2 S a^{2} \rho-E_{\theta} Y / 2 S a^{2} \rho(\lambda-$ $\left.\mu_{\theta}\right)$, and $T_{0}$ is the amplitude of the circular membrane. Next, we will solve the free vibration frequency of circular membrane in small deflection.

Substituting (29) into (9) yields

$$
\frac{d^{2} T(t)}{d t^{2}}+\left(-\frac{H_{1} \sigma_{0 r}}{S a^{2} \rho}\right) T(t)=0 .
$$

Equation (52) can be seen as (43) when $N=0$; substituting $N=0$ into (51) yields

$$
\omega=\frac{1}{a} \sqrt{\frac{\sigma_{0 r}}{\rho}} \cdot \sqrt{-\frac{H_{1}}{S}} .
$$

Directly letting $T_{0} \rightarrow 0$ in (51), the situation may degenerate into a small deflection. Equation (51) can be turned into the free vibration frequency of circular membrane in small deflection:

$$
\omega=\frac{1}{a} \sqrt{\frac{\sigma_{0 r}}{\rho}} \cdot \sqrt{-\frac{H_{1}}{S}} .
$$

So, the frequency formula which is deduced directly out according to the vibration of circular membrane in small deflection is equal to the frequency formula which is based on the vibration of circular membrane in large deflection. This proves that the result of this paper is correct.

\section{Computational Examples and Discussion}

Take the membrane material commonly applied in engineering as an example. Young's moduli are $E_{r}=1.4 \times 10^{6} \mathrm{kN} / \mathrm{m}^{2}$ and $E_{\theta}=0.9 \times 10^{6} \mathrm{kN} / \mathrm{m}^{2}$, respectively; Poisson's ratios are $\mu_{r}=0.3$ and $\mu_{\theta}=0.193$, respectively; the aerial density of the membrane materials is $\rho=1.7 \mathrm{~kg} / \mathrm{m}^{2}$; the circular membrane's thickness is $h=1.0 \mathrm{~mm}$. The first order vibration frequency of the membrane is calculated by (51).

We can draw the conclusion from the result of Table 1: The initial displacement (the amplitude) has influenced the vibration frequency of circular membrane on the basis of the large deflection theory. The frequency is enlarged with the increase of the initial displacement, and the larger the initial displacement is, the larger the effect on the frequency is, and vice versa. When the initial displacement approaches zero, the result is consistent with that obtained in this paper, according to the small deflection theory.

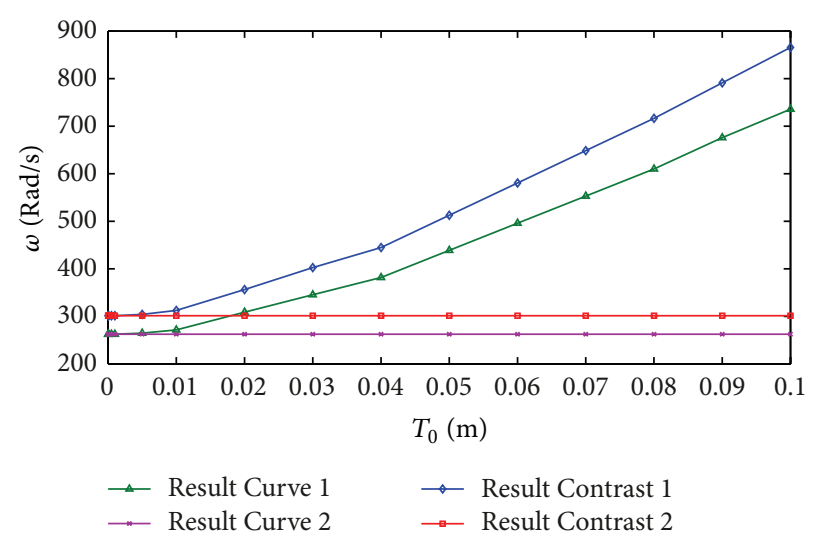

FIGURE 2: Frequency $(\omega)$-initial displacement $\left(T_{0}\right)$ curve.

In Figure 2, we have drawn the relation curve of frequency and initial displacement. "Result Curve 1" and "Result Contrast 1" are based on large deflection; "Result Curve 2" and "Result Contrast 2" are based on small deflection; "Result Contrast 1" and "Result Contrast 2" are the result for the other method which is used in [16]. It could be concluded that when the material properties and the initial pretension are given, there is nonlinear relationship between the vibration frequency and the initial deflection for the membrane in the large deflection; when the initial deflection $\left(T_{0}\right)$ is increasing, not only the vibration frequency is increasing, but also the slope of the curve will be slowly increasing, meaning $d \omega / d T_{0}$ is gradually increasing. This also shows that the larger $T_{0}$ is, the greater impact on $\omega$ it has. When the initial deflection approaches zero, the result is consistent with that obtained from the small deflection theory. Figure 2 reflects substantially the geometrical nonlinearity of the vibration of membranes; namely, formula (51) can reflect the geometric nonlinear characteristics of the vibration of membranes.

We can draw the conclusion from the comparative analysis of the relation curves of the frequency and the initial deflection drawn from this paper and [16]. The results of the two kinds of formula are consistent; they both reflect the nonlinear characteristics of the membrane vibration; therefore, the expression of frequency obtained in this paper is correct. In $[12,17]$, the Bessel equation was deduced, so using the Bessel function as the vibration function of the membrane is reasonable. The more the number of the expansions of the Bessel function is, the more accurate the frequency is. In practice, the number of the expansions is determined by necessity.

Compared to $[16,18,19]$, in this paper, more precise displacement potential function is applied, and the nonlinear vibration and orthotropy of membrane are taken into consideration too. In $[18,19]$, the thin circular plate is the object of study. In [18] the nonlinear vibration of thin circular plate has not been considered, and the initial pretension is imposed on the membrane in our paper, so it is different from [19]. In [16], the vibration displacement potential function of circular membrane is based on the vibration of the thin circular plate; its precision is not enough; the vibration of the plate and the 
TABLE 2: The frequency under various initial displacements and various pretensions $\left(\sigma_{o x}=\sigma_{o y}=\sigma_{\mathrm{o}}\right)$.

\begin{tabular}{lcccc}
\hline$T_{\mathrm{o}}(\mathrm{m})$ & 0.0022583 & 0.0023742 & 0.001593 & 0.0016005 \\
$\sigma_{o}\left(\mathrm{kN} / \mathrm{m}^{2}\right)$ & 1200 & 2400 & 3700 & 4800 \\
\hline$\omega_{1}(\mathrm{~Hz})$ & 13.2160 & 18.5630 & 22.9094 & 26.0790 \\
$\omega_{2}(\mathrm{~Hz})$ & 15 & 19 & 21 & 25 \\
\hline$T_{0}(\mathrm{~m})$ & 0.0015625 & 0.0014709 & 0.0013644 & 0.001361 \\
$\sigma_{o}\left(\mathrm{kN} / \mathrm{m}^{2}\right)$ & 6100 & 7300 & 8500 & 9800 \\
\hline$\omega_{1}(\mathrm{~Hz})$ & 29.3847 & 32.1331 & 34.6638 & 37.2162 \\
$\omega_{2}(\mathrm{~Hz})$ & 28 & 31 & 33 & 36 \\
\hline
\end{tabular}

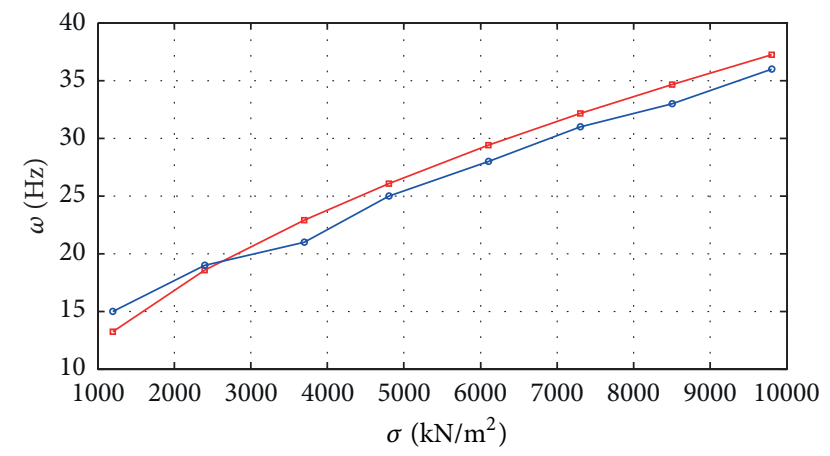

$\rightarrow \omega_{1}$

$\rightarrow \omega_{2}$

Figure 3: Frequency $(\omega)$-pretension $(\sigma)$ curve.

vibration of the thin membrane are different. In this paper, the Bessel function is chosen as the vibration displacement potential function of circular membrane; it is more precise.

This formula can also be used to solve other membrane structures with different geometry and boundary conditions. For example, assume a square membrane structure with $1.0 \mathrm{~m}$ in side length; then Young's moduli are $E_{x}=1.52 \times 10^{6} \mathrm{kN} / \mathrm{m}^{2}$ and $E_{y}=1.29 \times 10^{6} \mathrm{kN} / \mathrm{m}^{2}$, respectively; Poisson's ratios are $\mu_{x}=0.4$ and $\mu_{y}=0.39$, respectively; the aerial density of the membrane materials is $\rho=1.05 \mathrm{~kg} / \mathrm{m}^{2}$; the membrane's thickness is $h=0.82 \mathrm{~mm}$; the initial displacement is $T_{0}$. We used formula (51) to calculate the vibration frequency $\omega_{1}$, roughly. In Table 2 , the experimental results $\omega_{2}$ are given too.

In Figure 3, the calculation data is compared with the experimental data. The calculation results are consistent with the results obtained from the experiment.

\section{Conclusions}

We apply the large deflection theory of membrane and the principle of virtual displacement to derive the governing vibration equations of circular membrane with outer edges fixed, use the Bessel function to establish the accurate vibration mode function of the circular membrane, and then solve them by the Galerkin method. At last, we obtain the generally accurate formula of nonlinear vibration frequency of circular membrane with outer edges fixed. And the frequency of the vibration of circular membrane in small deflection which is degenerated by the vibration of circular membrane in large deflection is equal to the frequency which is deduced directly out, according to the vibration of circular membrane in small deflection. According to $[18,19]$, the formula of the vibration frequency for orthotropic membrane, which is based on the large deflection theory, is correct. The theoretical result is of great significance to study the dynamic response of membranes with forced vibration in large deflection and is also available to verify the correctness of the other numerical methods.

\section{Conflict of Interests}

The authors declare that there is no conflict of interests regarding the publication of this paper.

\section{Acknowledgment}

This research presented in this paper was supported by the National Natural Science Foundation of China (Project no. 51178485).

\section{References}

[1] S. Z. Shen, "A study on some theoretical aspects of long-span spatial structures," Journal of Suzhou Institute of Urban Construction and Environmental Protection, vol. 13, no. 3, pp. 1-8, 2000.

[2] H. S. Chang, "Development and strategy of reliability for longspan spatial," The World of Building Materials, vol. 33, no. 2, pp. 111-113, 2012.

[3] W. Z. Chien, H. S. Lin, H. C. Hu, and K. Y. Yeh, The Problem of Large Deflection of Elastic Circular Membranes, Science Press, Beijing China, 1953.

[4] R. Kao and N. Perrone, "Large deflections of axisymmetric circular membranes," International Journal of Solids and Structures, vol. 7, no. 12, pp. 1601-1612, 1971.

[5] C. J. Cheng and X. Yang, "The problem of large deflections of rotationally orthotropic circular membranes," Communication on Applied Mathematics and Computation, vol. 1, no. 1, pp. 3847, 1987.

[6] S.-L. Chen and Z.-L. Zheng, "Large deformation of circular membrane under the concentrated force," Applied Mathematics and Mechanics, vol. 24, no. 1, pp. 28-31, 2003.

[7] S. W. Kang, "Free vibration analysis of composite rectangular membranes with a bent interface," Journal of Sound and Vibration, vol. 272, no. 1-2, pp. 39-53, 2004.

[8] L. G. Young, S. Ramanathan, J. Hu, and P. F. Pai, "Numerical and experimental dynamic characteristics of thin-film membranes," International Journal of Solids and Structures, vol. 42, no. 9-10, pp. 3001-3025, 2005.

[9] R. M. Soares and P. B. Gonalves, "Nonlinear vibrations and instabilities of a stretched hyperelastic annular membrane," International Journal of Solids and Structures, vol. 49, no. 3-4, pp. 514-526, 2012.

[10] G. Z. Qian, "The solutions of the free vibration problem of planar membrane with two different direction of force," Applied Mathematics and Mechanics, vol. 3, no. 6, pp. 817-824, 1982. 
[11] X. Wu, "Natural vibration analysis of films with middle supports," Journal of Vibration and Shock, vol. 25, no. 1, pp. 140-141, 2006.

[12] W. J. Lin and S. H. Chen, "Free vibration analysis of annular membranes," Journal of Vibration and Shock, vol. 47, no. 2, pp. 103-108, 2008.

[13] C. Ou yang, "The solvability study for a vibrating membrane robin problem with perturbed boundary," Mathematics in Practice and Theory, vol. 39, no. 23, pp. 184-185, 2009.

[14] W. J. Lin and S. H. Chen, "Free vibration analysis of plane membranes by finite element method," Journal of Dynamics and Control, vol. 8, no. 3, pp. 202-206, 2010.

[15] L. Qiao, F. Tan, and Q.-S. Yang, "Dynamic analysis of membrane structures," Journal of Vibration and Shock, vol. 30, no. 6, pp. 109-113, 2011.

[16] Z. Zheng, W. Song, C. Liu, Y. Xu, and J. Long, "Free vibration analysis of orthotropic circular membranes in large deflection," Advanced Materials Research, vol. 255-260, pp. 1730-1734, 2011.

[17] W.-J. Lin and S.-H. Chen, "Free vibration analysis of circular membranes," Journal of Vibration and Shock, vol. 28, no. 5, pp. 84-86, 2009.

[18] J. J. Li, "Large deflection of orthotropic thin circular plates," Journal of Shanghai University (Natural Science Edition), vol. 3, no. 1, pp. 9-18, 1997.

[19] J. Wang, "Nonlinear free vibration of the circular plate with large deflection," Journal of South China University of Technology (Natural Science Edition), vol. 29, no. 8, pp. 4-6, 2001. 


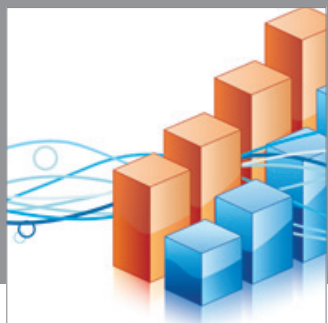

Advances in

Operations Research

mansans

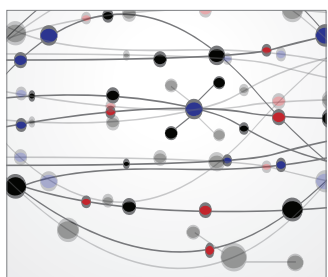

The Scientific World Journal
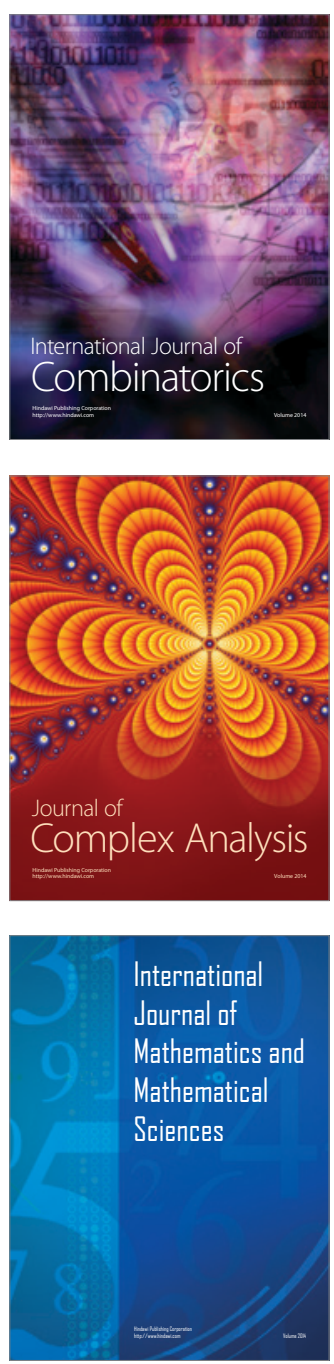
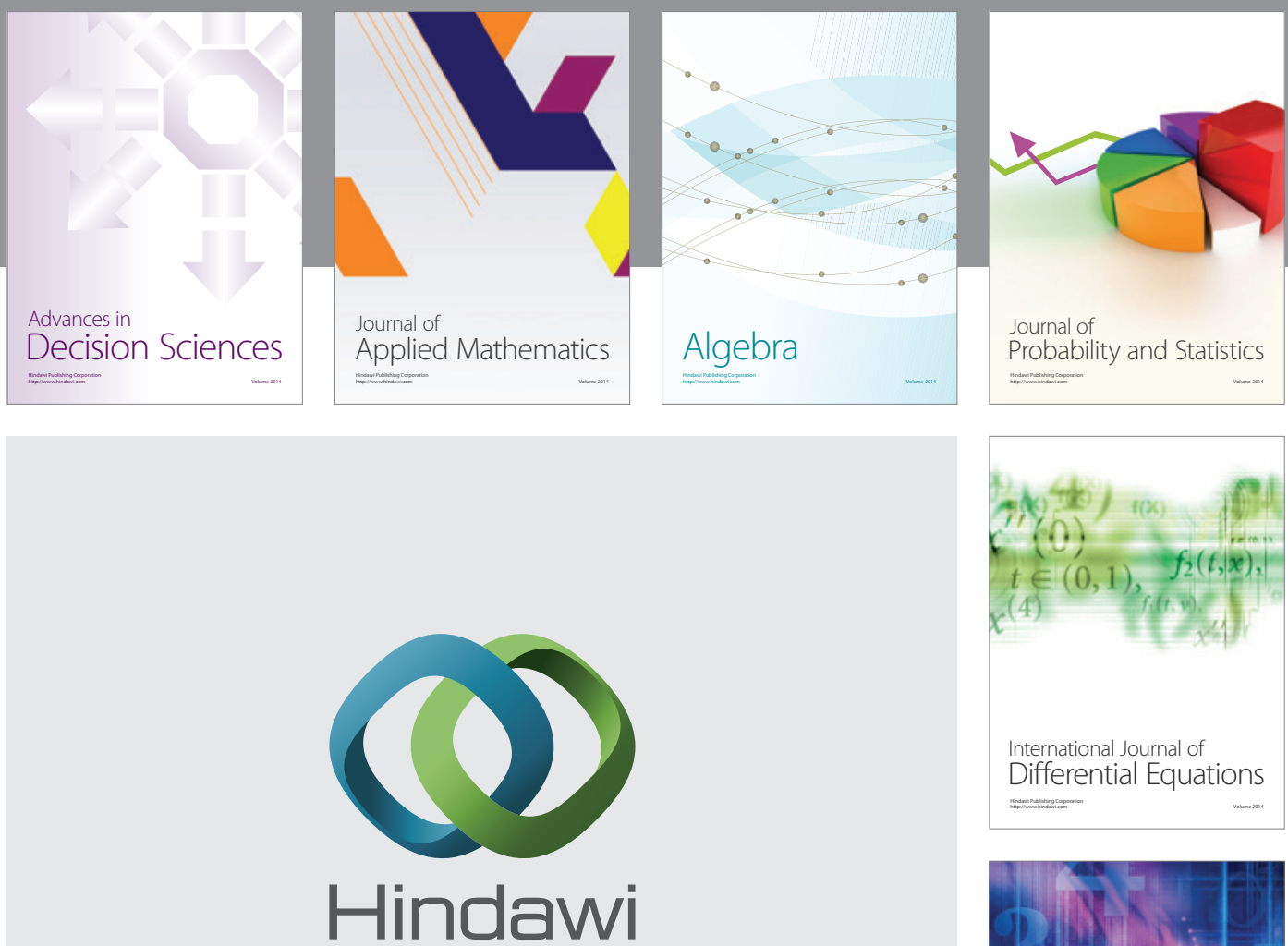

Submit your manuscripts at http://www.hindawi.com
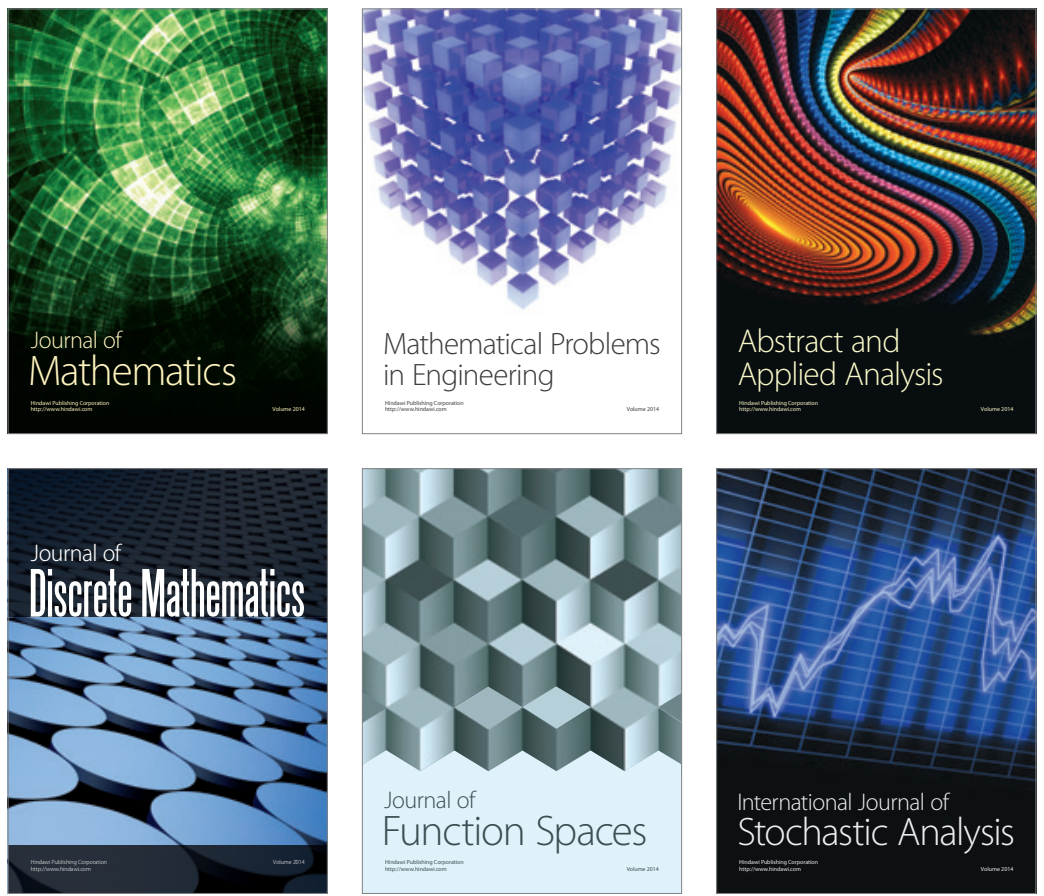

Journal of

Function Spaces

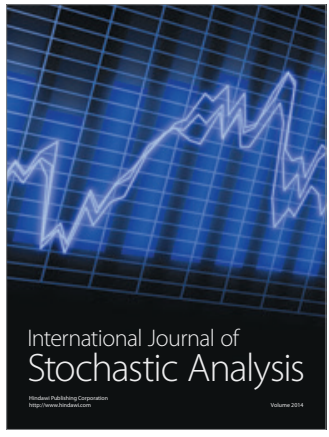

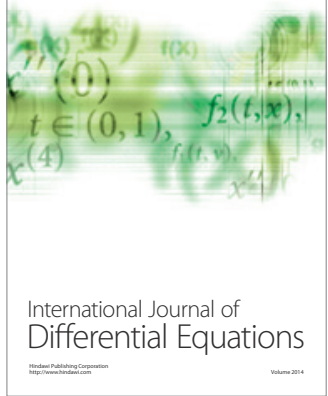
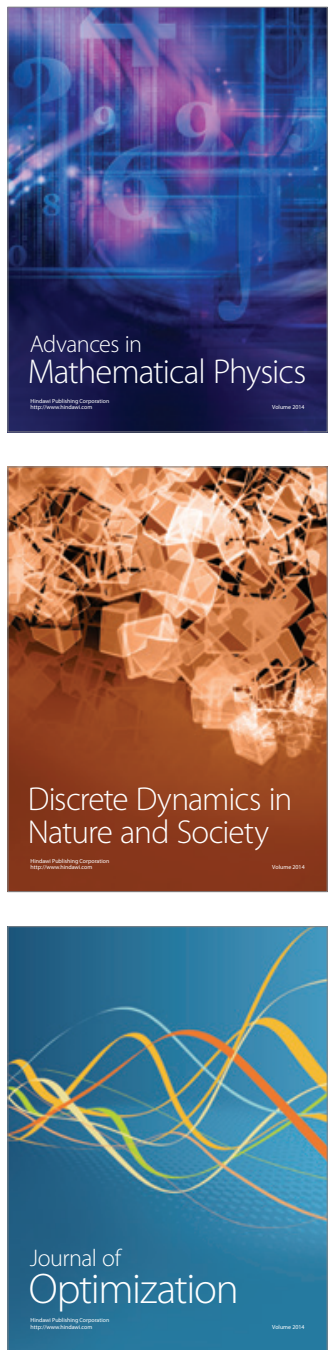